\title{
Determination of the Antiproton-to-Electron Mass Ratio by Precision Laser Spectroscopy of $\bar{p} \mathbf{H e}^{+}$
}

\author{
M. Hori, ${ }^{1,2}$ A. Dax, ${ }^{2}$ J. Eades, ${ }^{2}$ K. Gomikawa, ${ }^{2}$ R. S. Hayano, ${ }^{2}$ N. Ono, ${ }^{2}$ W. Pirkl, ${ }^{2}$ E. Widmann, ${ }^{3}$ H. A. Torii, ${ }^{4}$ \\ B. Juhász, ${ }^{5,3}$ D. Barna, ${ }^{6,2}$ and D. Horváth ${ }^{6}$ \\ ${ }^{1}$ CERN, CH-1211 Geneva 23, Switzerland \\ ${ }^{2}$ Department of Physics, University of Tokyo, 7-3-1 Hongo, Bunkyo-ku, Tokyo 113-0033, Japan \\ ${ }^{3}$ Stefan Meyer Institut für Subatomare Physik, Boltzmanngasse 3, Vienna 1090, Austria \\ ${ }^{4}$ Institute of Physics, University of Tokyo, Komaba, Meguro-ku, Tokyo 153-8902, Japan \\ ${ }^{5}$ Institute of Nuclear Research of the Hungarian Academy of Sciences, H-4001 Debrecen, Hungary \\ ${ }^{6}$ KFKI Research Institute for Particle and Nuclear Physics, H-1525 Budapest, Hungary
}

(Received 10 April 2006; published 19 June 2006)

\begin{abstract}
A femtosecond optical frequency comb and continuous-wave pulse-amplified laser were used to measure 12 transition frequencies of antiprotonic helium to fractional precisions of $(9-16) \times 10^{-9}$. One of these is between two states having microsecond-scale lifetimes hitherto unaccessible to our precision laser spectroscopy method. Comparisons with three-body QED calculations yielded an antiproton-to-electron mass ratio of $M_{\bar{p}} / m_{e}=1836.152674(5)$.
\end{abstract}

DOI: 10.1103/PhysRevLett.96.243401

We report here new measurements on the transition frequencies of antiprotonic helium atoms $\left(\bar{p} \mathrm{He}^{+} \equiv e^{-}-\right.$ $\bar{p}-{ }^{4} \mathrm{He}^{2+}$ ) [1] using a femtosecond optical frequency comb $[2,3]$ in conjunction with a continuous-wave (cw) pulse-amplified laser (Fig. 1). Their experimental precision is a factor 6-20 better than our previous best ones [4], and now approaches those of, e.g., the $1^{1} s-2^{1} s[5]$ and $1^{1} s-2^{1} p$ [6] transitions in ordinary helium. From the frequencies of 12 transitions measured to the Doppler-broadened limit at a cryogenic temperature of $10 \mathrm{~K}$, we have deduced the mass and charge of the antiproton relative to both the proton and the electron with a precision of the order of the known proton-to-electron mass ratio [7].

Reference [4] describes how a radio-frequency quadrupole decelerator was used to slow down the antiprotons emerging from the CERN Antiproton Decelerator to 100$\mathrm{keV}$ energies. They were then stopped in a helium target of low atomic density $\rho \sim 10^{18} \mathrm{~cm}^{-3}$ to produce $\bar{p} \mathrm{He}^{+}$ atoms which filled a volume $V \sim 100 \mathrm{~cm}^{3}$. Antiprotons in $\bar{p} \mathrm{He}^{+}$states with high principal $(n \sim 38)$ and angular momentum $(\ell)$ quantum numbers reach the helium nucleus over a period of several microseconds. The resulting delayed annihilation time spectra (DATS), i.e., the annihilation rate versus time elapsed since $\bar{p} \mathrm{He}^{+}$formation, was measured by Cherenkov counters [Fig. 2(a)]. In all but one of the present experiments, linearly polarized laser pulses of energy density $\varepsilon=0.04-1 \mathrm{~mJ} / \mathrm{cm}^{2}$ (e.g., applied here at $t \sim 1 \mu \mathrm{s})$ stimulated transitions with dipole moments 0.02-0.3 D from these $\bar{p} \mathrm{He}^{+}$states, to states with nanosecond-scale lifetimes against Auger emission [1] and annihilation. The resulting peak in the DATS signaled the resonant frequency.

Only pulsed lasers can provide the megawatt-scale intensities needed here to induce the $\bar{p} \mathrm{He}^{+}$transitions. However, fluctuations in their frequency and linewidth and the difficulty of calibrating the wide range of $\bar{p} \mathrm{He}^{+}$
PACS numbers: 36.10.-k, 06.20.Dk, 14.20.Dh, 32.70.Jz

wavelengths $\lambda=264.7-726.1 \mathrm{~nm}$ have so far limited our experimental precision [4]. We have now circumvented these problems by basing our experiments on a cw laser whose frequency $\nu_{\mathrm{cw}}$ could be stabilized with a precision $<4 \times 10^{-10}$ against an optical comb. Its intensity was then amplified $[6,8,9]$ by a factor $10^{6}$ to produce a pulsed laser beam of frequency $\nu_{\mathrm{pl}} \sim \nu_{\mathrm{cw}}$ with an accuracy and resolution 1-2 orders of magnitude higher than before [4].

This was done as follows: First, a $\mathrm{Nd}: \mathrm{YVO}_{4}$ laser (Coherent Verdi, B in Fig. 1) pumped either a ring Ti:sapphire or dye laser (Coherent MBR-110 or 899-21), thus producing $\mathrm{cw}$ laser beams covering the required wavelengths $\lambda=574.8-941.4 \mathrm{~nm}$ with linewidth $\Gamma=1$ or $4 \mathrm{MHz}$ and power $P \sim 1 \mathrm{~W}$. This seed beam was amplified in three dye cells pumped by a pulsed Nd:YAG laser (Coherent Infinity, C) of $\lambda=532 \mathrm{~nm}$, energy $E=$ $200 \mathrm{~mJ}$, and length $3 \mathrm{~ns}$. To decrease the Fourier-limited

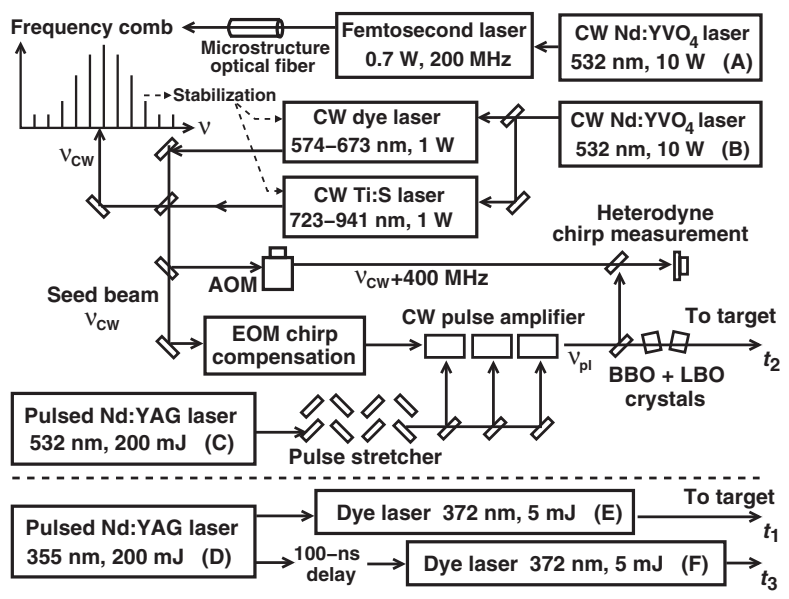

FIG. 1. Top: Stabilization of cw pulse-amplified laser to femtosecond frequency comb. Bottom: Additional dye lasers used to measure the 417.8-nm transition. 

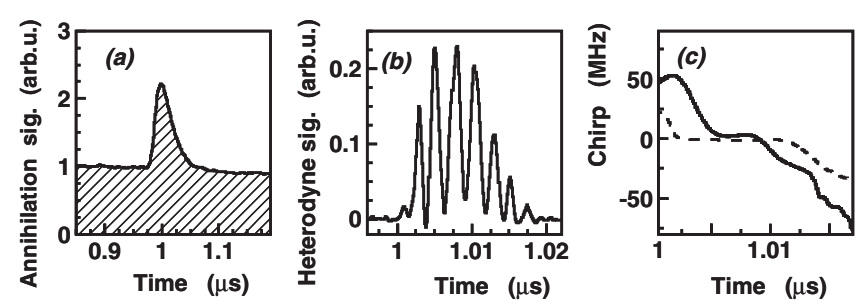

FIG. 2. (a) DATS of $\bar{p}^{4} \mathrm{He}^{+}$with laser-induced transition $(n, \ell)=(39,35) \rightarrow(38,34)$. (b) Heterodyne beat signal of the pulsed dye laser. (c) Time evolution of frequency chirp with (dashed line) and without (solid line) chirp compensation.

linewidth of the pulsed dye laser, the pump beam was split into seven beams, to which incremental delays were added. These were then merged to produce the stretched (20-nslong) pulse that irradiated each cell. The cells emitted laser pulses with $\Gamma \sim 60 \mathrm{MHz}$ and $E=5-20 \mathrm{~mJ}$. The shorter wavelengths $\lambda=264.7-470.7 \mathrm{~nm}$ were obtained by (i) frequency doubling the outputs at $574.8 \mathrm{~nm}$ and $728.8-$ $941.4 \mathrm{~nm}$ in, respectively, beta-barium borate (BBO) and lithium triborate (LBO) crystals, or (ii) frequency tripling the 794.1-nm light using both crystals.

The comb (Menlo Systems FC-8004) [2,3] constituted a mode-locked Ti:sapphire laser (Femtolasers Femtosource Scientific) pumped by a cw Nd: $\mathrm{YVO}_{4}$ laser (A in Fig. 1), which produced 15 -fs-long laser pulses of repetition rate $f_{\text {rep }}=200 \mathrm{MHz}$ and average power $P=0.7 \mathrm{~W}$. The spectral width $\lambda=750-850 \mathrm{~nm}$ of this pulsed beam was first broadened to $500-1100 \mathrm{~nm}$ by propagating it through a microstructure fiber. This beam was then used to stabilize (i) the frequency offset $f_{\text {off }}=20 \mathrm{MHz}$ common to all the modes of the comb [2,3] and (ii) the cw seed laser to frequency $\nu_{\mathrm{cw}}=n_{c} f_{\text {rep }}+f_{\text {off }}+f_{\text {dif }}$ which was $f_{\text {dif }}=$ $20 \mathrm{MHz}$ above the $n_{c}$ th mode of the comb. The value $n_{c}=$ 1592 190-2 607811 was measured using a Fizeau wavelength meter. All frequencies $f_{\text {rep }}, f_{\text {off }}$, and $f_{\text {dif }}$ were synchronized to a quartz oscillator, which was stabilized to a timing signal provided by global positioning satellites. The seed (and consequently the pulsed dye) laser was scanned over a region $\pm 4 \mathrm{GHz}$ around the $\bar{p} \mathrm{He}^{+}$lines by changing the above repetition rate from $f_{\text {rep }}=$ 200.000 to $200.004 \mathrm{MHz}$. Doppler-free spectroscopy of $\mathrm{Rb}$ and $\mathrm{I}_{2}$ in the seed beam indicated that its frequency precision was $<4 \times 10^{-10}$. A thermally stabilized, sealed housing permitted 24-h operation of the lasers during these months-long experiments. Motorized stages optimized the alignment and polarization of the beam coupled into the fiber during the 100-s intervals between antiproton pulses.

The frequency $\nu_{\mathrm{pl}}$ of the dye laser pulse can deviate from the seed value $\nu_{\mathrm{cw}}$ due to sudden changes in the refractive index of the dye during the amplification [6,9]. This socalled chirp effect of magnitude $\Delta \nu_{c}(t)=\nu_{\mathrm{pl}}(t)-\nu_{\mathrm{cw}}$ can shift the measured $\bar{p} \mathrm{He}^{+}$frequencies $\nu_{\exp }$ from their true values, so it had to be corrected. The time evolution of

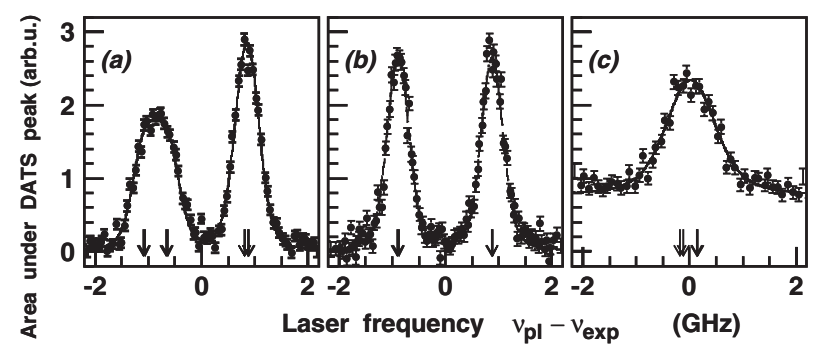

FIG. 3. Frequency profiles of the transition (a) $(36,34) \rightarrow(37,33)$ in $\bar{p}^{3} \mathrm{He}^{+}$, and (b) $(37,35) \rightarrow(38,34)$ and $(\mathrm{c})(36,34) \rightarrow(35,33)$ in $\bar{p}^{4} \mathrm{He}^{+}$(see text).

$\Delta \nu_{c}(t)$ was measured by (i) diverting part of the seed laser and shifting its frequency by $400 \mathrm{MHz}$ using an acoustooptic modulator (AOM), (ii) superimposing this beam of frequency $\nu_{\mathrm{cw}}+400 \mathrm{MHz}$ and the dye laser pulse on a photodiode, and recording their heterodyne beat signal [Fig. 2(b)] with a digital oscilloscope of bandwidth $f=$ $1.5 \mathrm{GHz}$ and sampling rate $8 \mathrm{GHz}$, and (iii) using Fourier analysis $[6,8]$ to isolate any frequency deviation of this signal from $400 \mathrm{MHz}$ caused by a chirp of value $\Delta \nu_{c}(t)$. In Fig. 2(c), the chirp in a 597.3-nm laser pulse which changed from $\Delta \nu_{c}(t)=50$ to $-70 \mathrm{MHz}$ over its 20 -ns duration is shown by the solid line. We minimized $\Delta \nu_{c}(t)$ (dashed line) using an electro-optic modulator (EOM) to apply a frequency shift of opposite sign to the seed laser, which canceled the chirp induced in the dye cells $[6,9,10]$. Theoretical calculations [11] show that any further shift in $\nu_{\text {exp }}$ caused by chirps induced in the BBO or LBO crystals is $<1-2 \mathrm{MHz}$.

The profile of the $(n, \ell)=(36,34) \rightarrow(37,33)$ resonance in $\bar{p}^{3} \mathrm{He}^{+}$(i.e., the area under the DATS peak plotted against the $\nu_{\mathrm{pl}}$ value) is shown in Fig. 3(a). It contains (i) eight intense lines (indicated by four arrowed pairs) corresponding to E1 transitions involving no spin-flip between the eight hyperfine substates [12] of states $(36,34)$ and $(37,33)$, and (ii) 12 weak lines wherein one of the constituent particles flips its spin. Only the two peaks separated by $1.8 \mathrm{GHz}$ that arise from the interaction between the orbital angular momentum of the antiproton and electron spin could be resolved, however, due to the 400$\mathrm{MHz}$ Doppler broadening caused by the motion of the
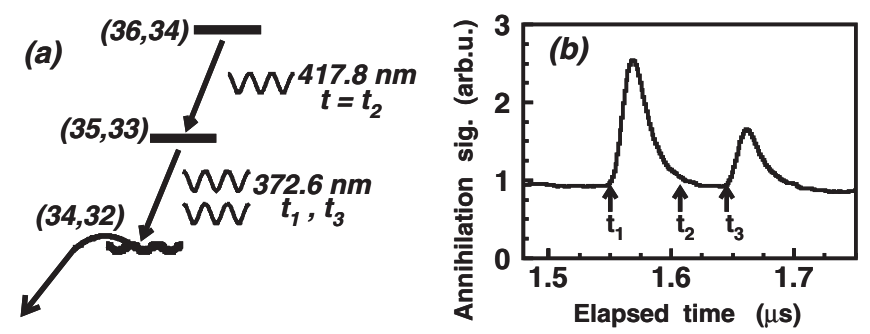

FIG. 4. (a) Portion of energy level diagram of $\bar{p}^{4} \mathrm{He}^{+}$. (b) DATS with three lasers fired at $t_{1}-t_{3}$ to measure the 417.8nm transition (see text). 
TABLE I. Transition frequencies of $\bar{p} \mathrm{He}^{+}$and its density shifts: experimental (with $1 \sigma$ errors) and theoretical values.

\begin{tabular}{ccccccc}
\hline \hline & Transition & \multicolumn{2}{c}{ Transition frequency (MHz) } & \multicolumn{2}{c}{ Density shift $\left(\times 10^{-18} \mathrm{MHzcm}^{3}\right)$} \\
& $(n, \ell) \rightarrow\left(n^{\prime}, \ell^{\prime}\right)$ & \multicolumn{1}{c}{ Experimental } & \multicolumn{1}{c}{ Korobov [15] } & Kino et al.[14] & Experimental & Bakalov et al.[17] \\
\hline $\bar{p}^{4} \mathrm{He}^{+}$ & $(40,35) \rightarrow(39,34)$ & $445608558(6)$ & 445608568.6 & 445608592 & $-8.3(3)$ & -6.45 \\
& $(39,35) \rightarrow(38,34)$ & $501948752(4)$ & 501948754.9 & 501948782 & $-4.2(2)$ & -3.96 \\
& $(37,35) \rightarrow(38,34)$ & $412885132(4)$ & 412885132.0 & 412885143 & $-3.8(2)$ & -3.22 \\
& $(37,34) \rightarrow(36,33)$ & $636878139(8)$ & 636878151.6 & 636878120 & $-1.4(1)$ & -1.39 \\
& $(36,34) \rightarrow(35,33)$ & $717474004(10)$ & 717474001.2 & 717473893 & & -0.59 \\
& $(35,33) \rightarrow(34,32)$ & $804633059(8)$ & 804633058.1 & 804633026 & $-0.4(1)$ & -0.21 \\
& $(32,31) \rightarrow(31,30)$ & $1132609209(15)$ & 1132609223.5 & 1132609194 & & 0.41 \\
$\bar{p}^{3} \mathrm{He}^{+}$ & $(38,34) \rightarrow(37,33)$ & $505222296(8)$ & 505222280.1 & 505222260 & $-4.1(2)$ & -4.58 \\
& $(36,34) \rightarrow(37,33)$ & $414147508(4)$ & 414147508.0 & 414147512 & $-4.0(2)$ & -3.83 \\
& $(36,33) \rightarrow(35,32)$ & $646180434(12)$ & 646180407.7 & 646180389 & $-1.7(1)$ & -1.61 \\
& $(34,32) \rightarrow(33,31)$ & $822809190(12)$ & 822809170.7 & 822809199 & $-0.4(1)$ & -0.34 \\
& $(32,31) \rightarrow(31,30)$ & $1043128609(13)$ & 1043128579.6 & 1043128649 & $0.3(1)$ & 0.26 \\
\hline \hline
\end{tabular}

$\bar{p}^{3} \mathrm{He}^{+}$thermalized to $T=10 \mathrm{~K}$. The spin-averaged transition frequency $\nu_{\exp }$ was determined by fitting this profile with the theoretical line shape (solid line) obtained from the optical Bloch equations which describe the evolution of the $\bar{p} \mathrm{He}^{+}$state populations during laser irradiation. The small remaining chirp [e.g., similar in magnitude to the dashed line in Fig. 2(c)] introduced a time dependence to $\nu_{\mathrm{pl}}(t)$ when performing the Bloch equation integration. In this we took transitions between all hyperfine and magnetic substates into account, using the theoretical values for their splittings (precision $<1 \mathrm{MHz}$ ) and dipole moments [12]. Doppler broadening, laser power broadening, and collisional dephasing effects were also included. The $\nu_{\exp }$ values of $\bar{p}^{4} \mathrm{He}^{+}$resonances [Fig. 3(b)], which contain four intense, non-spin-flip lines [indicated by arrowed pairs as in Fig. 3(c)] and four weak, spin-flip ones were similarly obtained. The ac Stark shifts caused by the laser interacting with $\bar{p} \mathrm{He}^{+}$are estimated to be $\ll 1 \mathrm{MHz}$, due to the small scalar ( -3 to 2 a.u.) and tensor $\left[(0.1-2) \times 10^{-3}\right.$ a.u. $]$ terms of the dynamic polarizability for these transitions [13].

All transitions heretofore accessible to our precision laser spectroscopy involved a daughter state with a short Auger lifetime, the natural width $\Gamma_{n} \geq 20 \mathrm{MHz}[14,15]$ of which would ultimately limit the achievable precision on $\nu_{\exp }$ to around $\sim 10^{-9}$. We have now extended our studies to include one $\bar{p}^{4} \mathrm{He}^{+}$transition $(36,34) \rightarrow(35,33)$ between two metastable states with $\Gamma_{n} \sim 100 \mathrm{kHz}$. This implies an ultimate precision of $\sim 10^{-12}$, although our present experiments are Doppler rather than natural-width limited. To measure this transition we developed the following three-laser method [Fig. 4(a)], which also utilizes the above cw pulse-amplified laser: (i) An additional dye laser (Lambda Physik Scanmate, E in Fig. 1) pumped by a 355-nm Nd:YAG laser (D) first irradiated the $\bar{p} \mathrm{He}^{+}$with a 3 -ns-long pulse at $\lambda=372.6 \mathrm{~nm}$. This depleted the population in state $(35,33)$ at $t_{1}$ by inducing the transition $(35,33) \rightarrow(34,32)$ to a short-lived state, and thereby producing the first peak in Fig. 4(b). (ii) At $t=t_{2}$, the cw pulse-amplified laser tuned to $(36,34) \rightarrow(35,33)$ at $\lambda=$ $417.8 \mathrm{~nm}$ equalized the population in the parent and daughter states. (iii) Another 372.6-nm dye laser (F) pumped by the same Nd:YAG laser after a 100-ns-delay probed the increased population of $(35,33)$ at $t=t_{3}$ resulting from the transitions stimulated by the 417.8-nm laser pulse, and produced an annihilation peak at $t=t_{3}$. The profile of the $(36,34) \rightarrow(35,33)$ resonance obtained by plotting the intensity of this peak against the frequency of the 417.8-nm laser is shown in Fig. 3(c).

Collisions between $\bar{p} \mathrm{He}^{+}$and helium atoms cause the transition frequencies $\nu$ to shift linearly with target density
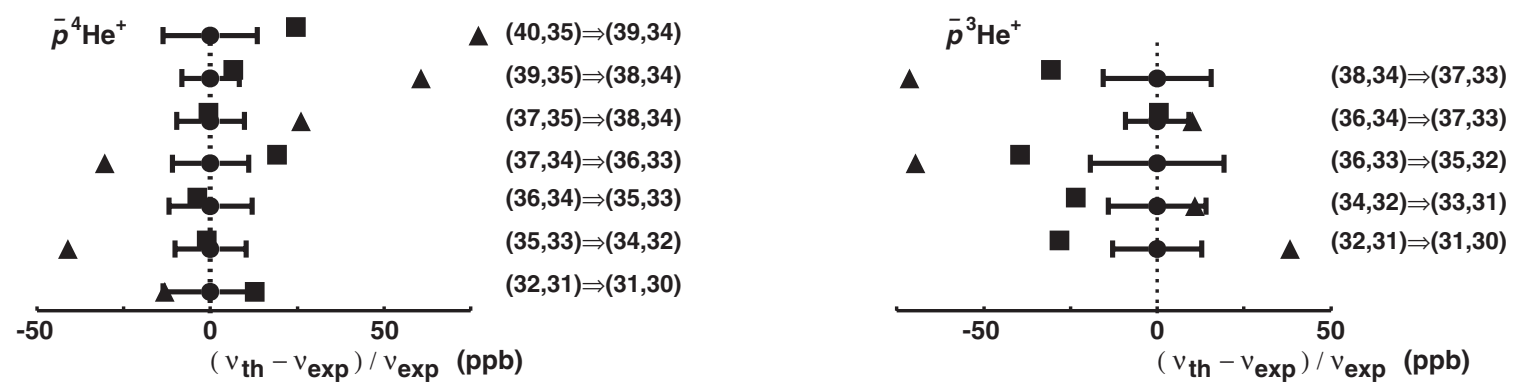

FIG. 5. Experimental $\nu_{\text {exp }}$ (circles with $1 \sigma$ errors) vs theoretical $\nu_{\text {th }}$ (triangles [14] and squares [15]) transition frequencies. 


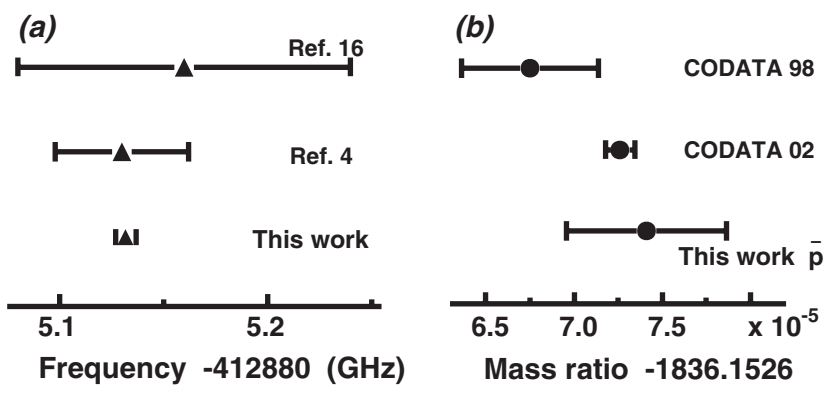

FIG. 6. (a) Frequency of the $\bar{p}^{4} \mathrm{He}^{+}$transition $(37,35) \rightarrow$ $(38,34)$ measured in this and previous $[4,16]$ experiments. (b) Proton-to-electron [7] and antiproton-to-electron mass ratios.

[16]. We measured the shifts in ten transitions using the above Scanmate dye lasers at densities $\rho=2 \times 10^{18}-3 \times$ $10^{21} \mathrm{~cm}^{-3}$, and determined the gradients $d \nu / d \rho$ (Table I). The results agreed with $a b$ inito quantum chemical calculations [17]. The in vacuo values $\nu_{\exp }(\rho=0)$ were obtained by correcting the experimental results using a shift between $\Delta \nu=-14$ and $1 \mathrm{MHz}$ corresponding to density $\rho \sim 2 \times 10^{18} \mathrm{~cm}^{-3}$. The experimental 1 standard deviation $(1 \sigma)$ error $\sigma_{\text {exp }}=4-15 \mathrm{MHz}$ (Fig. 5) was the quadratic sum of the statistical one associated with the above fitting (3-13 MHz), and systematic ones $\sigma_{\text {syst }}$ arising from the chirp (2-4 MHz), collisional shifts $(0.1-2 \mathrm{MHz})$, and the harmonic generation (1-2 MHz).

The $\nu_{\exp }$ values agree with previous experiments $[4,16]$ within the order-of-magnitude lower precision of the latter [Fig. 6(a)]. They are compared with two sets of theoretical values $\nu_{\text {th }}[14,15]$ in Fig. 5, both of which include QED and nuclear-size $\left(\Delta E_{\text {nuc }}=2-4 \mathrm{MHz}\right)$ effects. Values from Ref. [14] scatter within 7-8 $\sigma$ of $\nu_{\text {exp }}$. Those of Ref. [15], the only calculation claiming precision $\sigma_{\text {th }}=1-2 \mathrm{MHz}$ commensurate with $\sigma_{\text {exp }}$, agree within $<1 \times 10^{-8}$ with the four highest-precision measurements in $\bar{p}^{4} \mathrm{He}^{+}$, and $(36,34) \rightarrow(37,33)$ in $\bar{p}^{3} \mathrm{He}^{+}$. Four of its $\bar{p}^{3} \mathrm{He}^{+}$frequencies were $\sim 2 \sigma$ below our $\nu_{\exp }$ values. Concerning Ref. [14], unpublished results from the authors have recently moved by 3-100 MHz from those in Table I. We therefore use only the Ref. [15] values in the following.

The mass ratios between the proton, ${ }^{4} \mathrm{He}$ and ${ }^{3} \mathrm{He}$ nuclei, and electron $M_{p} / m_{e}=1836.15267261(85), M_{\alpha} / m_{e}=$ 7294.2995363(32), and $M_{{ }^{3} \mathrm{He}} / m_{e}=5495.885269(11)$ [7] and other Committee on Data for Science and Technology (CODATA) 2002 constants were first used [15] to calculate $\nu_{\text {th }}$ (Table I). These ratios increased by 3-6 ppb between the 1998 and 2002 compilations due to improved measurements of $m_{e}$ and $M^{3_{3}} \mathrm{He}$ [7]. We next determined the antiproton-to-electron mass ratio as the value $M_{\bar{p}} / m_{e}=1836.152674(5)$ which results in $\nu_{\text {th }}\left(M_{\bar{p}} / m_{e}\right)$ values that agree best with experiment, i.e., which minimizes the sum $\Sigma\left[\nu_{\text {th }}\left(M_{\bar{p}} / m_{e}\right)-\nu_{\exp }\right]^{2} / \sigma_{\exp }^{2}$ over the 12 transitions. This is consistent with the above proton value [Fig. 6(b)]. Here $\sigma_{\exp }$ is mostly statistical. When we changed $M_{\bar{p}} / m_{e}$ by $3 \mathrm{ppb}$, the resulting $3-5 \mathrm{MHz}$ change in $\nu_{\text {th }}$ was comparable with $\sigma_{\text {exp }}$. The error 5 on the last digit of $M_{\bar{p}} / m_{e}$ is the quadratic sum of 4 (the minimization error) and the systematic ones 3 (arising from $\sigma_{\text {syst }}$ ) and 2 (from $\sigma_{\text {th }}$ ). We can also set a new upper limit $\delta_{\bar{p}}$ on any $C P T$-violating difference [18] between the antiproton and proton charges $\left(Q_{\bar{p}}, Q_{p}\right)$ and masses using the values of $\nu_{\text {th }}$ and $\nu_{\text {exp }}$ in Table I and the equation $[1,4] \delta_{\bar{p}}=\left(Q_{p}+\right.$ $\left.Q_{\bar{p}}\right) / Q_{p} \sim\left(M_{p}-M_{\bar{p}}\right) / M_{p}=\left(\nu_{\text {th }}-\nu_{\exp }\right) / \nu_{\exp } \kappa$. Values for $\kappa=2.5-6.1$ were estimated [14] by increasing $Q_{\bar{p}}$ and $M_{\bar{p}}$ by $1 \mathrm{ppm}$ under the constraint on $Q_{\bar{p}} / M_{\bar{p}}$ against $Q_{p} / M_{p}$ of $<9 \times 10^{-11}$ [19] and noting the change in $\nu_{\mathrm{th}}$. The average value of $\delta_{\bar{p}}$ over 12 transitions was $(-3 \pm$ 14) $\times 10^{-10}$, which implies that the antiproton charge and mass agree with the proton's to a precision of $2 \times 10^{-9}$ at a confidence level of $90 \%$. The precisions of these determinations were partially limited by the differences between $\nu_{\text {th }}$ and $\nu_{\text {exp }}$ in $\bar{p}^{3} \mathrm{He}^{+}$.

We thank D. Bakalov, Y. Kino, and V.I. Korobov for theoretical discussions, and K. S. E. Eikema, M. Fischer, R. Holzwarth, and K. Jungmann for invaluable expertise and advice concerning the lasers. This work was supported by Monbukagakusho (Grant No. 15002005), and the Hungarian Scientific Research Fund (OTKA T046095).

[1] T. Yamazaki et al., Phys. Rep. 366, 183 (2002).

[2] Th. Udem, R. Holzwarth, and T. W. Hänsch, Nature (London) 416, 233 (2002).

[3] D. J. Jones et al., Science 288, 635 (2000).

[4] M. Hori et al., Phys. Rev. Lett. 91, 123401 (2003).

[5] S. D. Bergeson et al., Phys. Rev. Lett. 80, 3475 (1998).

[6] K.S. E. Eikema et al., Phys. Rev. Lett. 76, 1216 (1996); Phys. Rev. A 55, 1866 (1997).

[7] P. J. Mohr and B. N. Taylor, Rev. Mod. Phys. 72, 351 (2000); 77, 1 (2005).

[8] M.S. Fee, K. Danzmann, and S. Chu, Phys. Rev. A 45, 4911 (1992).

[9] I. Reinhard et al., Appl. Phys. B 63, 467 (1996).

[10] V. Meyer et al., Phys. Rev. Lett. 84, 1136 (2000).

[11] A. V. Smith and M. S. Bowers, J. Opt. Soc. Am. B 12, 49 (1995).

[12] V. I. Korobov, Phys. Rev. A 73, 022509 (2006).

[13] V.I. Korobov (private communication).

[14] Y. Kino, H. Kudo, and M. Kamimura, Mod. Phys. Lett. A 18, 388 (2003); (private communication).

[15] V. I. Korobov, in Proceedings of the EXA05 International Conference on Exotic Atoms and Related Topics, edited by A. Hirtl, J. Marton, E. Widmann, and J. Zmeskal (Austrian Academy of Sciences Press, Vienna, 2005).

[16] M. Hori et al., Phys. Rev. Lett. 87, 093401 (2001).

[17] D. Bakalov et al., Phys. Rev. Lett. 84, 2350 (2000).

[18] R. J. Hughes and B. I. Deutch, Phys. Rev. Lett. 69, 578 (1992).

[19] G. Gabrielse et al., Phys. Rev. Lett. 82, 3198 (1999). 